\title{
Stability Improvement of Two Wheel Driven Mobile Manipulator Using Nonlinear PD Controller
}

\author{
Pradeep K.W. Abeygunawardhana Student Member (Keio University, kumara@sum.sd.keio.ac.jp) \\ Murakami ToshiyukiＭember (Keio University, mura@sd.keio.ac.jp)
}

Keywords: two wheel mobile manipulator, double inverted pendulum, inverted pendulum, disturbance observer, acceleration control, nonlinear PD controller

Nonlinear control can exploit where parameter uncertainties and complexity of choosing correct gains are present. Mobile manipulation offers a dual advantage of mobility offered by the platform and dexterity offered by the manipulator. This paper proposes the stability improvement of two wheeled mobile manipulator using nonlinear Proportional Derivative (PD) controller. Nonlinear PD controller is a controller which automatically adjusts its gains according to the stability conditions on running. Lyapunov function based on kinetic energy of the system and position error is used to re-schedule the gains of the nonlinear PD controller. This method will expand the stability margin of the two wheeled mobile manipulator. Altitude control of two wheeled mobile manipulator was achieved by using virtual inverted pendulum. Modeling of virtual double inverted pendulum using body and 3 manipulator link is shown in Fig. 1.

$M_{w}, M_{1}$ and $M_{2}$ are masses of the wheel, body and pendulum. $R$ is radius of the wheel and $l_{0}$ is distance between centre of gravity of body and wheel center. $l_{d}$ is length of the pendulum. Lagrange equation of motion is used to derive the dynamic equations. After linearization system becomes as described in (1), (2) and (3).

$$
\begin{aligned}
\tau_{s} & =M_{d} \ddot{q}+k_{d} q \ldots \ldots \ldots \ldots \ldots \ldots \ldots \ldots \ldots \ldots \ldots \ldots \ldots \ldots \\
M_{d} & =\left[\begin{array}{ccc}
M_{w}+M_{1}+M_{2} & \left(M_{1}+M_{2}\right) l_{0} & M_{2} l_{d} \\
\left(M_{1}+M_{2}\right) l_{0} & \left(M_{1}+M_{2}\right) l_{0}^{2} & M_{2} l_{0} l_{d} \\
M_{2} l_{d} & M_{2} l_{0} l_{d} & M_{2} l_{d}^{2}
\end{array}\right] \ldots \ldots \\
k_{d} & =\left[\begin{array}{ccc}
0 & 0 & 0 \\
0 & -\left(M_{1}+M_{2}\right) g l_{0} & 0 \\
0 & 0 & -M_{2} g l_{d}
\end{array}\right] \ldots \ldots \ldots \ldots \ldots
\end{aligned}
$$

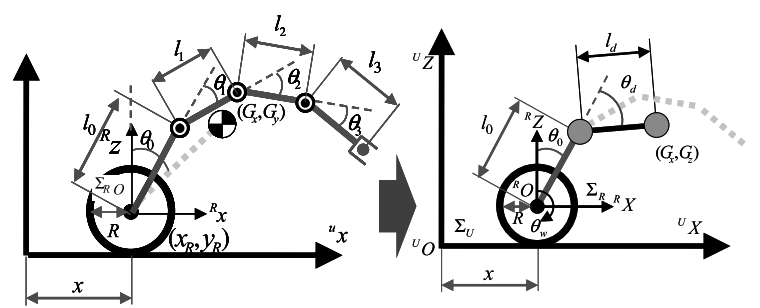

Fig. 1. Double inverted pendulum model
Dynamic equations of it can be simplified as given in (4) and (5).

$$
\begin{aligned}
& \ddot{\theta}_{0}=-\frac{1}{l_{0}}\left(\ddot{x}+\frac{M_{2} l_{d} \ddot{\theta}_{d}}{M_{1}+M_{2}}+g \theta_{0}\right) \\
& \ddot{x}=R \ddot{\theta}_{w} \ldots \ldots \ldots \ldots \ldots \ldots \ldots \ldots \ldots \ldots \ldots \ldots
\end{aligned}
$$

In order to stabilize the (4), (6) is approximated. Position command and velocity command of wheel are calculated by integrating (6).

$$
\begin{aligned}
\ddot{\theta}_{w}^{c m d}= & \frac{l_{0}}{R}\left\{K_{p d}\left(\theta_{0}^{r e s}-\theta_{0}^{c m d}\right)+K_{v d}\left(\dot{\theta}_{0}^{r e s}-\dot{\theta}_{0}^{c m d}\right)\right. \\
& \left.-\frac{M_{2} l_{d} \ddot{\theta}_{d}}{\left(M_{1}+M_{2}\right)}\right\} \ldots \ldots \ldots \ldots \ldots \ldots \ldots \ldots
\end{aligned}
$$

$K_{p d}$ is the position gain of wheel command estimation and $K_{v d}$ is the velocity gain of wheel command estimation. Acceleration reference of the wheel motor is calculated as shown in (7).

$$
\ddot{\theta}_{w}^{\text {ref }}=\left(K_{p}\left(\theta_{w}-\theta_{w}^{\text {res }}\right)+K_{v}\left(\dot{\theta}_{w}-\dot{\theta}_{w}^{\text {res }}\right)\right)
$$

Gains are designed as given in (8) and (9) with linear and nonlinear terms. $V$ is the function which depends on stability of the system and it is given in (10)

$$
\begin{array}{r}
K_{p}=K_{0 p}+\frac{K_{1 p}}{2}\left(1+e^{-\alpha V}\right) \ldots \ldots \ldots \ldots \\
K_{v}=2 \zeta_{w} \sqrt{K_{p}} \ldots \ldots \ldots \ldots \ldots \ldots \ldots \\
V\left(\dot{q}, \dot{\theta}_{w}, e_{r}\right)=\frac{1}{2} \dot{q}^{T} M(q) \dot{q}+\frac{1}{2} \dot{\theta}_{w}^{T} M n \dot{\theta}_{w} \\
+\frac{1}{2} e_{r}^{T} K_{D} e_{r} \ldots \ldots \ldots \ldots
\end{array}
$$

where $e_{r}=\left(x^{\text {cmd }}-x\right)$ and $K_{D}$ is the positive definitive diagonal matrix. Three links manipulator is controlled to achieved desired pendulum position in workspace. Manipulator controller consists of workspace controller and null space controller. Nonlinear controller is designed at workspace of pendulum. Experiment was carried out and result shows the proposed method is effective. 


\title{
Stability Improvement of Two Wheel Driven Mobile Manipulator Using Nonlinear PD Controller
}

\author{
Pradeep K. W. Abeygunawardhana* Student Member \\ Toshiyuki Murakami* Member
}

\begin{abstract}
This paper proposes the stability improvement of two wheeled mobile manipulator using nonlinear Proportional Derivative (PD) controller. Nonlinear PD controller is a controller which automatically adjusts its gains according to the stability conditions on running. Lyapunov function based on kinetic energy of the system and position error are used to re-schedule the gains of the nonlinear PD controller. This method will expand the stability margin of the two wheeled mobile manipulator. Further advantages of this method are reducing the parameter uncertainty and difficulty of adjusting gains due to nonlinear nature of system. In the proposed approach, virtual double inverted pendulum is introduced for the control of the two wheeled mobile manipulator. The model based on the double inverted pendulum, makes it easy to design the controller. Here, wheel control and manipulator control are designed separately and unified by using two nonlinear PD controllers. Two nonlinear PD controllers are implemented for better stability. The method proposed in this paper has used nonlinear PD controller in work space of the manipulator controller together with null space control. Here the null space control increases the control stability of manipulator configuration. The effectiveness of the proposed method was confirmed by the experiment of two wheel mobile manipulator.
\end{abstract}

Keywords: two wheel mobile manipulator, double inverted pendulum, inverted pendulum, disturbance observer, acceleration control, nonlinear PD controller

\section{Introduction}

High positioning accuracy and low tracking error, in both position and velocity are important performance indices in robotics and precision motion control. On the other hand, the desire of having a better performance behavior of the control system has led to the development of many sophisticated control algorithms/methods ${ }^{(1)}$. However, in spite of performance limitation, PD (Proportional and Derivative) controller is most widely used for robot positioning control because of its simplicity. More advanced controllers such as computed torque or resolved acceleration control are often incorporated with PD control ${ }^{(2)(3)}$.

Nonlinear Proportional and Derivative (PD) control has been proposed by improving the performance of PD control. The main advantages of nonlinear PD control are its high disturbance rejection and robustness to the time delay ${ }^{(2)(4)}$.

Mobile manipulation provides dual advantage of mobility and dexterity offered by the platform and the manipulator respectively. The degree of freedom of the platform is also added the redundancy of the system. These systems can be effectively used for a variety of tasks such as industrial robots, service robotics etc. Two wheel mobile manipulator has the ability to balance on its two wheels and spin on the spot. This additional maneuverability allows easy navigation on various terrains, turn sharp corners and traverse small steps or curbs.

The inverted pendulum problem is not uncommon in the

\footnotetext{
* Keio University

3-14-1, Hiyoshi, Kouhoku-ku, Yokohama 223-8522
}

field of control engineering. The uniqueness and wide application of technology derived from this unstable system have drawn the interest of many researchers and robotic enthusiasts around the world ${ }^{(11)-(13)}$. In recent years, they have applied the idea of a mobile inverted pendulum model to various activities like designing walking gaits for humanoid robots, robotic wheelchairs and personal transport systems. A scaled down prototype of a Digital Signal Processor controlled two wheel vehicle is a product of the Industrial Electronics Laboratory at the Swiss Federal Institute of Technology. It is based on the inverted pendulum, with weights attached to the system known as JOE ${ }^{(5)}$. Another improvement of the research world is balancing of bicycle robots which can run without a rider and with wheel architecture different from above systems ${ }^{(6)(7)}$.

Two wheel driven mobile manipulator has been proposed using the virtual inverted pendulum control in which the error is relatively large ${ }^{(8)}$. The passivity based control of two wheel mobile manipulator is also reported ${ }^{(9)}$. Although, they have proposed improvement of null space control, range of stability margin has not been adequate.

This paper proposes stability improvement of two wheel mobile manipulator using nonlinear PD control. The control algorithm presented here is a kind of adaptive control. The two original contributions of this paper are the application of nonlinear PD control with the inverted pendulum control and the use of nonlinear PD control for work space of manipulator and wheels, while it is controlled in null space. It is also hard to find the nonlinear PD control with mobile robot and important robot applications. Fig. 1 depicts the two wheel mobile robot. 


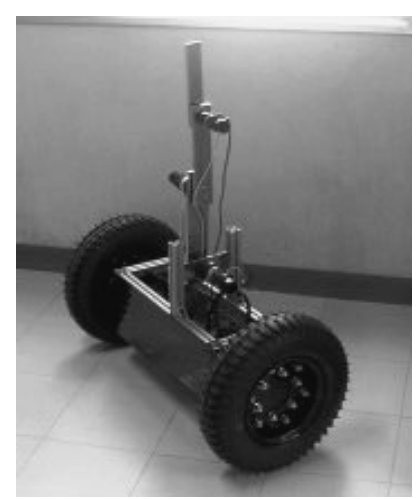

Fig. 1. Two wheel vehicle

This paper presents in eight sections. First Introduction and section 2 describes Modeling of robot. Section 3 explains designing procedure of wheel command. Section 4 describes posture control using Centre Of Gravity (COG) control. Section 5 is the most important section of this paper and it explains the designing of nonlinear PD controller. Sections 6, 7 and 8 are control system, Experiment results and conclusion respectively.

\section{Modeling}

Modeling is described in this section. The two wheel robot has coaxial two wheel system. A direct Current (DC) servo motor with a encoder which is used to measure wheel angles, has been mounted to a wheel. Vehicle body with three link manipulator has assembled to wheel system. Each manipulator link is controlled using a DC servo motor with encoder. The body has been connected to the wheel system via a passive joint. The angle of passive joint was measured using an inclination sensor. The body connected through the passive joint, is also assumed as a manipulator link. Therefore, the robot is assumed as a two wheel robot with four degree of freedom manipulator.

2.1 Dynamics Dynamic modeling is described in this section. The model of the mobile manipulator is shown in Fig. 2. Variables that are used in modeling process are given below.

$\theta_{i} \quad \mathrm{i}=0$ Inclination angle of the vehicle body

$\mathrm{i}=1,2,3$ Joint angle of link

$i=4$ Rotation angle of wheel

$l_{0} \quad$ Distance between wheel axle and joint 1

$l_{n} \quad$ the length of the nth link

$m_{n}$ the mass of the nth link

$R \quad$ Radius of wheel

$\Sigma_{u} \quad$ Universal coordinate

$\Sigma_{R} \quad$ Robot coordinate

$\theta_{0}$ is the passive joint of this mechanism. Manipulator is working with $\mathrm{X}-\mathrm{Z}$ plane. Horizontal plane represents by the $\mathrm{X}$-plane and vertical plane represents the Z-plane. $x_{R}, z_{R}$ are robots coordinates. Clockwise rotation of vehicle body, wheels and all links are considered to be positive. In this model, dynamic equations are formulated using Lagrange equation of motion. The Lagrange equation is shown in (1).

$$
\frac{d}{d t}\left(\frac{\partial L}{\partial \dot{\theta}_{i}}\right)-\frac{\partial L}{\partial \theta_{i}}=\tau_{i},
$$

$n=1,2,3$. $i$ represents the joint number and $n$ is the num-

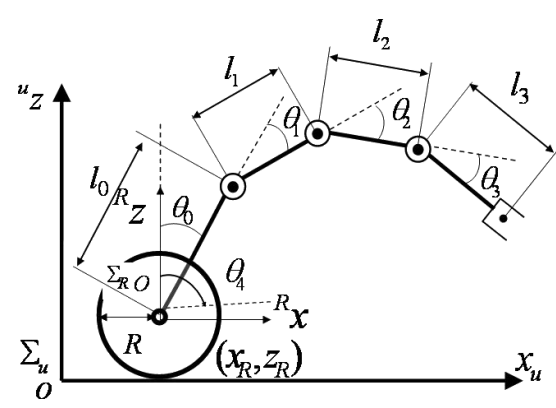

Fig. 2. Two wheel vehicle

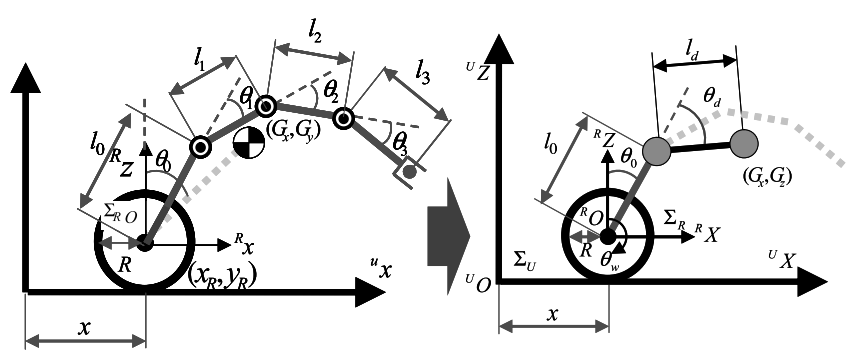

Fig. 3. Double inverted pendulum model

ber of joints. $L$ is called Lagrangian function and is given by $L=T-U$. T is the kinetic energy of the system and $\mathrm{U}$ is the potential energy of the system.

Deriving the kinetic energy and the potential energy of the system and simplifying with (1), dynamic equation of the system can be obtained as (2).

$$
\tau=\boldsymbol{M} \ddot{\boldsymbol{\theta}}+\boldsymbol{H}+\boldsymbol{G}
$$

where, $\tau$ is the vector of torques generated of joint motors. $M$ is inertia matrix. $H$ represents Coriolis acceleration coefficient and centrifugal acceleration coefficient. $G$ represents gravity term.

2.2 Double Inverted Pendulum Model of double inverted pendulum is shown in Fig. 3. Here $l_{d}$ is calculated as given in (3).

$$
l_{d}=\sqrt{\left(G_{x}-l_{0} S_{0}\right)^{2}+\left(G_{y}-l_{0} C_{0}\right)^{2}} .
$$

In Fig. 3 and (3), the explanation of each parameter is summarized below.

$$
\begin{array}{ll}
G_{x} & \mathrm{x} \text { distance of COG } \\
G_{y} & \mathrm{y} \text { distance of COG } \\
l_{d} & \text { Distance between wheel axel and COG } \\
\theta_{d} & \text { Angle of second pendulum }
\end{array}
$$

$G_{x}$ and $G_{y}$ are given as follows.

$$
\begin{gathered}
G_{x}=\frac{m_{1} l_{0} S_{0}+m_{2} l_{1} S_{01}+m_{3} l_{2} S_{012}+m_{4} l_{3} S_{0123}}{m_{1}+m_{2}+m_{3}+m_{4}} \\
\ldots \ldots \ldots \ldots \ldots \ldots \ldots \ldots \ldots \ldots \ldots \\
G_{y}=\frac{m_{1} l_{0} C_{0}+m_{2} l_{1} C_{01}+m_{3} l_{2} C_{012}+m_{4} l_{3} C_{0123}}{m_{1}+m_{2}+m_{3}+m_{4}}
\end{gathered}
$$

Abbreviations are defined as $S_{01}=\sin \left(\theta_{0}+\theta_{1}\right)$ and $C_{01}=$ $\cos \left(\theta_{0}+\theta_{1}\right)$. Inclination angle of the virtual inverted pendulum is obtained as indicated in (6). 


$$
\theta_{d}=\sin ^{-1}\left(\frac{G_{x}-l_{0} S_{0}}{l_{d}}\right)-\theta_{0}
$$

Equation (2) becomes as (7) in case of double inverted pendulum.

$$
\boldsymbol{\tau}_{\boldsymbol{d}}=\boldsymbol{M}_{d}(\boldsymbol{q}) \ddot{\boldsymbol{q}}+\boldsymbol{h}_{d}(\boldsymbol{q}, \dot{\boldsymbol{q}})+\boldsymbol{g}_{d}(\boldsymbol{q})
$$

Where, $\boldsymbol{q}=\left[x, \theta_{0}, \theta_{d}\right]^{T} \boldsymbol{\tau}_{d}=[F, 0,0]^{T}$

$\boldsymbol{M}_{d}(\boldsymbol{q})$ is a symmetric matrix and its element is given by,

$$
m_{d 11}=\left(M_{w}+M_{1}+M_{2}\right) \quad m_{d 12}=\left(M_{1}+M_{2}\right) l_{0} \cos \theta_{0}
$$$$
m_{d 13}=M_{2} l_{d} \cos \theta_{d} \quad m_{d 22}=\left(M_{1}+M_{2}\right) l_{0}^{2}
$$$$
m_{d 23}=M_{2} l_{0} l_{d} \cos \left(\theta_{0}-\theta_{d}\right) \quad m_{d 33}=M_{2} l_{d}^{2}
$$

$$
\begin{gathered}
\boldsymbol{h}_{d}=\left[\begin{array}{c}
-\left(M_{1}+M_{2}\right) l_{0} \sin \left(\theta_{0}\right) \dot{\theta}_{0}{ }^{2}-M_{2} l_{d} \sin \left(\theta_{d}\right) \dot{\theta}_{d}^{2} \\
M_{2} l_{0} l_{d} \sin \left(\theta_{0}-\theta_{d}\right) \dot{\theta}_{0} \dot{\theta}_{d} \\
M_{2} l_{0} l_{d} \sin \left(\theta_{0}-\theta_{d}\right) \dot{\theta}_{0}^{2} \\
\ldots \ldots \ldots \ldots \ldots \ldots \ldots \ldots \ldots \ldots
\end{array}\right] \\
\boldsymbol{g}_{d}=\left[\begin{array}{c}
0 \\
0 \\
\left(M_{1}+M_{2}\right) g l_{0} \sin \left(\theta_{0}\right) \\
M_{2} g l_{d} \sin \left(\theta_{d}\right)
\end{array}\right] \ldots \ldots \ldots \ldots \ldots \ldots
\end{gathered}
$$

Here $M_{w}$ is the mass of wheels $M_{1}$ is the mass of Body and $M_{2}$ is the mass of manipulators.

\section{Wheel Command Design}

Joint between wheel axel and the robot body is a passive joint. Therefore torque can not be modeled. Hence the only way of controlling this system is the controlling of wheel angles.

Stability margin of double inverted pendulum is larger than that of single inverted pendulum. The former is used in this paper. Dynamic equations of it can be simplified as given in (10) and (11).

$$
\begin{aligned}
& \ddot{\theta}_{0}=-\frac{1}{l_{0}}\left(\ddot{x}+\frac{M_{2} l_{d} \ddot{\theta}_{d}}{M_{1}+M_{2}}+g \theta_{0}\right) \\
& \ddot{x}=R \ddot{\theta}_{w} \ldots \ldots \ldots \ldots \ldots \ldots \ldots \ldots \ldots \ldots \ldots
\end{aligned}
$$

In order to stabilize the (10), (12) is approximated. By substituting the (12) into (10), equation (15) can be derived. Acceleration command to the wheel motor is calculated refering to (16)

$$
\begin{aligned}
\ddot{\theta}_{w}^{c m d}= & \frac{l_{0}}{R}\left\{K_{p d}\left(\theta_{0}^{r e s}-\theta_{0}^{c m d}\right)+K_{v d}\left(\dot{\theta}_{0}^{r e s}-\dot{\theta}_{0}^{c m d}\right)\right. \\
& \left.-\frac{M_{2} l_{d} \ddot{\theta}_{d}}{\left(M_{1}+M_{2}\right)}\right\} \ldots \ldots \ldots \ldots \ldots \ldots \ldots
\end{aligned}
$$

$K_{p d}$ is the position gain of wheel command estimation and $K_{v d}$ is the velocity gain of wheel command estimation. Angular position command and the angular velocity command are generated by integrating the angular acceleration command as given in (13) and (14)

$$
\begin{aligned}
\dot{\theta}_{w}^{c m d} & =\int \ddot{\theta}_{w}^{c m d} d t \\
\theta_{w}^{c m d} & =\int \dot{\theta}_{w}^{c m d} d t
\end{aligned}
$$

Here, it is assumed that the disturbance imposed on the wheel is suppressed by the disturbance observer. This brings wheel acceleration controller shown in (16).

$$
\begin{array}{r}
\ddot{\theta}_{0}^{r e f}=K_{v d}\left(\dot{\theta}_{0}^{c m d}-\dot{\theta}_{0}\right)+K_{p d}\left(\theta_{0}^{c m d}-\theta_{0}\right)+g \theta_{0} \\
\ldots \ldots \ldots \ldots \ldots \ldots \\
\ddot{\theta}_{w}=\ddot{\theta}_{w}^{c m d}, \quad \dot{\theta_{w}}=\dot{\theta}_{w}^{c m d}, \quad \theta_{w}=\theta_{w}^{c m d} \ldots \ldots \ldots
\end{array}
$$

Using equation (15), $G(s)$ can be obtained.

$$
\begin{aligned}
G(s) & =\frac{\theta_{0}}{\theta_{0}^{c m d}} \\
& =\frac{K_{p d}+K_{v d} s}{s^{2}+K_{v d} s+K_{p d}+g} .
\end{aligned}
$$

Natural angular frequency, $\omega_{n}$ and damping coefficient, $\zeta$ are given in (18) and (19) and those are selected as system become stable.

$$
\begin{aligned}
& \omega_{n}=\sqrt{K_{p d}+g} \\
& \zeta=\frac{K_{v d}}{2 \omega_{n}} \ldots \ldots
\end{aligned}
$$

\section{Posture Control Using COG Control}

In $n$ link manipulator, kinematics relationship can be expressed as shown in (20)-(22). $\boldsymbol{q}$ is the joint angle vector and is defined by $\boldsymbol{q}=\left(\theta_{0}, \theta_{1}, \theta_{2}, \theta_{3}\right)$ and $\boldsymbol{x}$ is the position vector in work space.

$$
\begin{aligned}
\boldsymbol{x} & =f(\boldsymbol{q}) \\
\dot{\boldsymbol{x}} & =\boldsymbol{J}(\boldsymbol{q}) \dot{\boldsymbol{q}} \cdots \ldots \\
\ddot{\boldsymbol{x}} & =\boldsymbol{J}(\boldsymbol{q}) \ddot{\boldsymbol{q}}+\boldsymbol{J}(\boldsymbol{q}) \dot{\boldsymbol{q}}
\end{aligned}
$$

where $\boldsymbol{J}$ is Jacobian matrix. $f(\boldsymbol{q})$ is described in equation (23) and abbreviations used here are as follows. $S_{01}=$ $\sin \left(\theta_{0}+\theta_{1}\right), C_{01}=\cos \left(\theta_{0}+\theta_{1}\right)$.

$$
\begin{aligned}
f(\boldsymbol{q}) & =\left[\begin{array}{l}
f_{1}(\boldsymbol{q}) \\
f_{2}(\boldsymbol{q})
\end{array}\right] \ldots \ldots \ldots \ldots \ldots \ldots \ldots \ldots \ldots \\
& =\left[\begin{array}{l}
l_{0} S_{0}+l_{1} S_{01}+l_{2} S_{012}+l_{3} S_{0123} \\
l_{0} C_{0}+l_{1} C_{01}+l_{2} C_{012}+l_{3} C_{0123}
\end{array}\right] \ldots \ldots
\end{aligned}
$$

In the redundant manipulator, joint space acceleration reference which was obtained using pseudo inverse matrix, can be written as (25). In (25), first term is the work space acceleration and second term represents the null space motion.

$$
\ddot{\boldsymbol{q}}^{r e f}=\boldsymbol{J}^{+}\left(\ddot{\boldsymbol{x}}^{r e f}-\dot{\boldsymbol{J}}(\boldsymbol{q}) \dot{\boldsymbol{q}}\right)+\left(\boldsymbol{I}-\boldsymbol{J}^{+} \boldsymbol{J}\right) \ddot{\boldsymbol{\phi}}
$$

where, $\boldsymbol{J}^{+}$is the weighted pseudo inverse matrix and defined as (26).

$$
\boldsymbol{J}^{+}=W^{-1}\left(\boldsymbol{J}^{T} \boldsymbol{J} W^{-1} \boldsymbol{J}^{T}\right)^{-1}
$$

In the above equation $\boldsymbol{W}$ is a diagonal weighting matrix. In the joint space disturbance observer based acceleration controller, $\boldsymbol{W}$ corresponds virtual inertia matrix $\boldsymbol{I}_{v n}$ and can be selected arbitrary ${ }^{(14)}$. By using the joint space and workspace observer, (25) can be rewritten as (27) without calculating the $\dot{\boldsymbol{J}}(\boldsymbol{q}) \dot{\boldsymbol{q}}$ and $\left(\boldsymbol{I}-\boldsymbol{J}^{+} \boldsymbol{J}\right)$ terms $^{(15)}$.

$$
\ddot{\boldsymbol{q}}^{\text {ref }}=\boldsymbol{J}^{+} \ddot{\boldsymbol{x}}^{r e f}+\ddot{\boldsymbol{q}}_{\text {null }}^{r e f} \text {. }
$$

\section{Nonlinear PD Controller Design}

Intuitively, two wheel mobile manipulator is an underactuated mechanical system due to the passive joint. Certain 


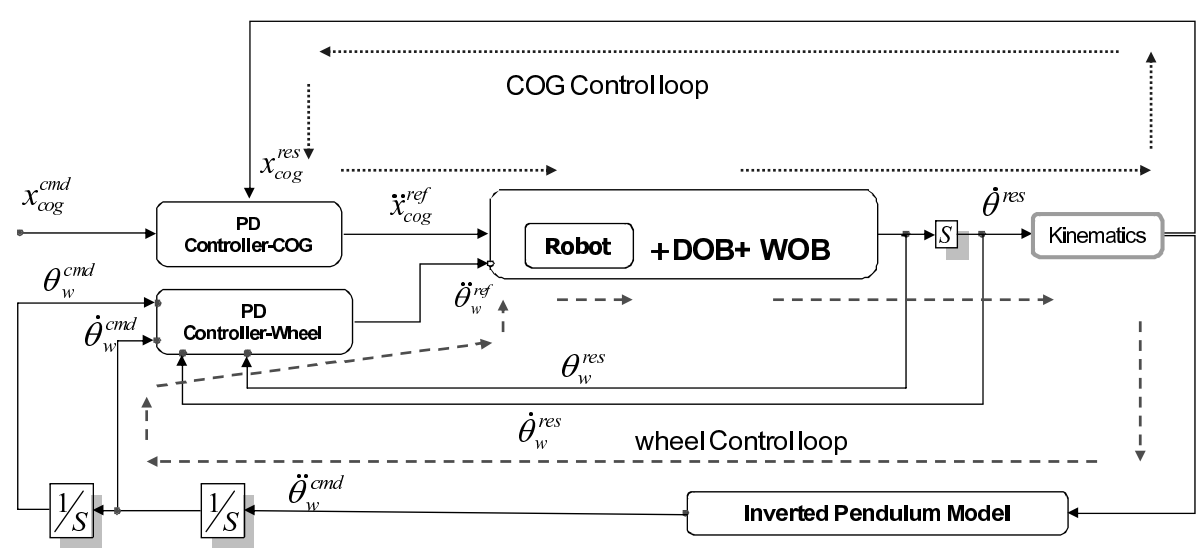

Fig. 4. Two control modes

design conditions have to be imposed for better stability of such a system ${ }^{(16)}$. This paper proposes nonlinear PD controller to improve the performance of two wheel mobile manipulator. In our approach, several approximations are made. And also it is difficult to get the precise estimations of the terms $\frac{M_{2} l_{d} \ddot{\theta}_{d}}{\left(M_{1}+M_{2}\right)}$ and $g \theta_{0}$ due to sensor trade off and modeling errors. Hence nonlinear controllers are proposed to improve the system stability.

5.1 Mode Decomposition Controller of this system has two parts known as manipulator controller and wheel controller. According to two controllers, system is decomposed into two modes as trajectory mode and balancing mode. Fig. 4 shows the simplified block diagram of total control system and two modes. Those are illustrated as "COG control loop" and "Wheel control loop" which represent the trajectory mode and the balancing mode.

In the trajectory mode, main purpose of the controller is to achieve correct trajectory. That is, high priority is given to the wheel control and controller increases the input signal due to trajectory error by switching the control gains of wheel PD controller into higher values.

In the balancing mode, priority is given to balance the robot body. Position gain and velocity gain of manipulator controller are switched to higher values. In this approach when one controller increases its gains, gains of other controller are decreased.

5.2 Controller Design Position error and velocity error based controller gains design method has already been proposed $^{(2)(4)}$. However, mobile manipulator has two subsystems and two nonlinear controllers have to be designed. And the value of switching function should be changed according to stability of the system. Therefore switching function should be determined by considering dynamic properties of both subsystems. It is designed using kinetic energy of the system and position error of workspace and given by (28).

$$
\begin{aligned}
V\left(\dot{q}, \dot{\theta}_{w}, e_{r}\right)= & \frac{1}{2} \dot{q}^{T} M(q) \dot{q}+\frac{1}{2} \dot{\theta}_{w}^{T} M n \dot{\theta}_{w} \\
& +\frac{1}{2} e_{r}^{T} K_{D} e_{r} \ldots \ldots \ldots \ldots
\end{aligned}
$$

where $e_{r}=\left(x^{c m d}-x\right)$ and $K_{D}$ is the positive definitive diagonal matrix.

5.2.1 Wheel Nonlinear Controller Position command, $\theta_{w}^{c m d}$ and velocity command, $\dot{\theta}_{w}^{c m d}$ that derived using pendulum model are used to generate the acceleration reference and those are shown in (16). Gains are designed in two parts as linear part and nonlinear part. Linear acceleration reference is selected as (29).

$$
\ddot{\theta}_{w}^{r e f}=\left(K_{0 p}\left(\theta_{w}-\theta_{w}^{r e s}\right)+K_{0 v}\left(\dot{\theta}_{w}-\dot{\theta}_{w}^{r e s}\right)\right)
$$

Taking Laplace transform of (29) and simplifying, equation (30) is derived.

$$
G(s)=\frac{2 \zeta_{w} \omega_{w n} s+\omega_{w n}^{2}}{s^{2}+2 \zeta_{w} \omega_{w n} s+\omega_{w n}^{2}}
$$

Characteristics of the second order equation given by (30), are natural angular frequency $\left(\omega_{w n}\right)$ and damping coefficient $\left(\zeta_{w}\right)$. Therefore $K_{0 p}$ and $K_{0 v}$ are selected as (31).

$$
\begin{aligned}
& K_{0 p}=\omega_{w n}^{2} \\
& K_{0 v}=2 \zeta_{w} \sqrt{K_{0 p}} .
\end{aligned}
$$

However, it is difficult to find correct gains due to nonlinearity of the system. Therefore, position gain is modified by adding nonlinear term as given in (32). Modified velocity gain can be calculated as (33).

$$
\begin{aligned}
K_{p} & =K_{0 p}+\frac{K_{1 p}}{2}\left(1+e^{-\alpha V}\right) \\
K_{v} & =2 \zeta_{w} \sqrt{K_{p}} \ldots \ldots \ldots \ldots \ldots
\end{aligned}
$$

First term of the right hand side of (32), $K_{0 p}$ is a constant term. $\alpha$ is a constant value and $K_{1 p}$ is the maximum change of position gain. Second term of the (32) is nonlinear and it is changed according to the function $V\left(\dot{q}, \dot{\theta}_{w}, e_{r}\right)$. If the system is going to be out of balance, $V\left(\dot{q}, \dot{\theta}_{w}, e_{r}\right)$ is increasing. Nonlinear part is designed to decrease the position gain with the increase of $V\left(\dot{q}, \dot{\theta}_{w}, e_{r}\right) . K_{1 p}$ should be selected carefully to avoid shifting out the stability. With the modified gains, final acceleration reference to the wheel motor is shown in (34).

$$
\ddot{\theta}_{w}^{r e f}=\left(K_{p}\left(\theta_{w}-\theta_{w}^{r e s}\right)+K_{v}\left(\dot{\theta}_{w}-\dot{\theta}_{w}^{r e s}\right)\right)
$$

5.2.2 Manipulator Nonlinear Controller Reference acceleration value at joint space of manipulator which is given by (27) is the sum of work space acceleration and null space acceleration reference. Work space acceleration value of manipulator in linear mode is calculated as (35).

$$
\ddot{\boldsymbol{x}}^{r e f}=K_{0 h p}\left(\boldsymbol{x}^{c m d}-\boldsymbol{x}\right)+K_{0 h v}\left(\dot{\boldsymbol{x}}^{c m d}-\dot{\boldsymbol{x}}\right) \cdot
$$


Taking Laplace transform of (35) and simplifying, equation (36) is derived.

$$
G(s)=\frac{2 \zeta_{m} \omega_{m n} s+\omega_{m n}^{2}}{s^{2}+2 \zeta_{m} \omega_{m n} s+\omega_{m n}^{2}}
$$

$\omega_{m n}$ and $\zeta_{m}$ are natural angular frequency and damping coefficient of the equation (36). Therefore $K_{0 h p}$ and $K_{0 h v}$ are selected as (37).

$$
\begin{aligned}
& K_{0 h p}=\omega_{m n}^{2} \\
& K_{0 h v}=2 \zeta_{m} \sqrt{K_{0 p}}
\end{aligned}
$$

Proportional gain and derivative gain are modified by adding the nonlinear terms and are given by (38) and (39).

$$
\begin{aligned}
K_{h p} & =K_{0 h p}+\frac{2 K_{1 h p}}{\left(1+e^{-\alpha V}\right)} \\
K_{h v} & =2 \zeta_{m} \sqrt{K_{h p}} \ldots \ldots \ldots
\end{aligned}
$$

$K_{0 h p}$ is constant value. $K_{1 h p}$ is the maximum shift of position gain and $\alpha$ a is the positive constant. Gains of manipulator control decrease as the value of function, $V\left(\dot{q}_{1}, \dot{\theta}_{w}, e_{r}\right)$ increases. After calculating nonlinear gains, work space acceleration reference value is calculated as (40).

$$
\ddot{\boldsymbol{x}}^{r e f}=K_{h p}\left(\boldsymbol{x}^{c m d}-\boldsymbol{x}\right)+K_{h v}\left(\dot{\boldsymbol{x}}^{c m d}-\dot{\boldsymbol{x}}\right)
$$

Fig. 5 depicts the expected variation of position gains in both modes. Null space reference acceleration value is selected as shown in (41). First and second term of the right hand side guarantees the Liyapunov stability. $K_{2}$ is the positive gain matrix.

$$
\ddot{\boldsymbol{q}}_{\text {null }}^{r e f}=J_{\text {aco }}^{T} K_{D}\left(x^{c m d}-x\right)+g(q)-K_{2} \dot{\boldsymbol{q}}
$$

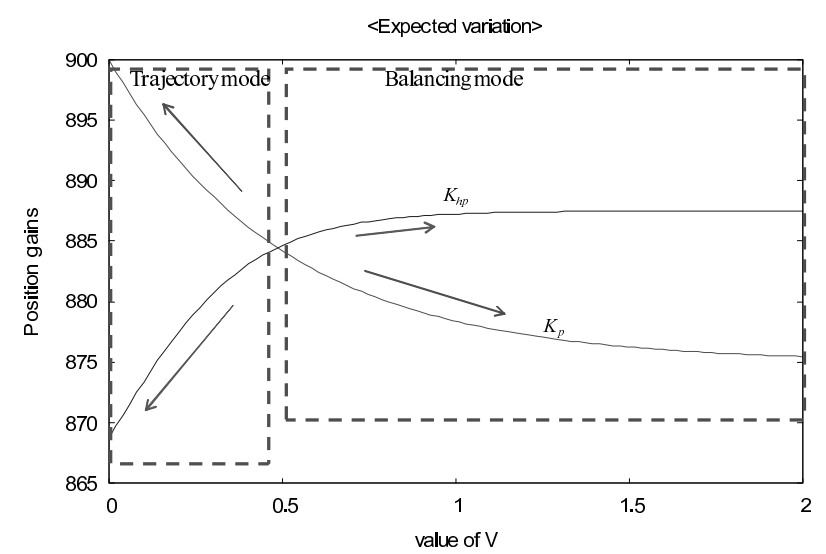

5.2.3 Liyapunov Stability Function $V\left(\dot{q}, \dot{\theta}_{w}, e_{r}\right)$ is positive define function. Differentiating the (28) along the trajectory and simplification result the (42). Property of skew symmetry was used in this simplification ${ }^{(19)}$.

$$
\begin{aligned}
\dot{V}\left(\dot{q}, \dot{\theta}_{w}, e_{r}\right)= & \dot{q}^{T}\left(\tau-J_{a c o}^{T} K_{D} e_{r}-g(q)\right) \\
& +\dot{\theta}_{w}^{T} M n \ddot{\theta}_{w} \ldots \ldots \ldots \ldots \\
= & \dot{q}^{T}\left(\tau-J_{a c o}^{T} K_{D} e_{r}-g(q)\right) \\
& +\dot{\theta}_{w}^{T} \tau_{w} \ldots \ldots \ldots \ldots \ldots
\end{aligned}
$$

Global convergence of the proposed method was assured by selecting the inputs for joint torques and wheel torque as given in (44) and (45).

$$
\begin{aligned}
& \tau=J_{a c o}^{T} K_{D}\left(x^{c m d}-x\right)+g(q)-K_{2} \dot{\boldsymbol{q}} \\
& \tau_{w}=-K_{1} \dot{\theta}_{w} \ldots \ldots \ldots \ldots \ldots \ldots \ldots
\end{aligned}
$$

where $K_{1}$ is positive define diagonal matrix. If the equations (44) and (45) were selected and $K_{1}$ is large enough, then the existence of relationship (46) is assured.

$$
\dot{V}\left(\dot{q}, \dot{\theta}_{w}, e_{r}\right) \leq-\dot{\theta}_{w}^{T} K_{1} \dot{\theta}_{w}-\dot{\boldsymbol{q}}^{T} K_{2} \dot{\boldsymbol{q}}
$$

$V\left(\dot{q}, \dot{\theta}_{w}, e_{r}\right)$ is positive definite function and $\dot{V}\left(\dot{q}, \dot{\theta}_{w}, e_{r}\right)$ is negative semi definite. Further $V\left(\dot{q}, \dot{\theta}_{w}, e_{r}\right)$ is bounded. That is, $V\left(\dot{q}, \dot{\theta}_{w}, e_{r}\right)$ become infinite as variables become infinite. Hence, system is stable according to the Liyapunov function used here.

\section{Control System}

Robot has two subsystems known as manipulator system and wheel system. Accordingly, control system also consists of wheel controller and manipulator controller. Two controllers are put together by means of virtual pendulum model for the stability of whole system. Fig. 6 depicts the simplified diagram of full control system. According to the desired COG position command, three link manipulator moves and controls the position of the virtual inverted pendulum. Acceleration reference at joint space is generated by nonlinear PD controller and null space controller. Disturbance observer cancels the disturbances of manipulator motors and work space observer corrects the error at work space. Kinematics represents the conversion of joint angle into position vector.

Fig. 7 illustrates the control system of manipulator control in detail. Implementations of null space and nonlinear PD

Fig. 5. Expected variations of control gains

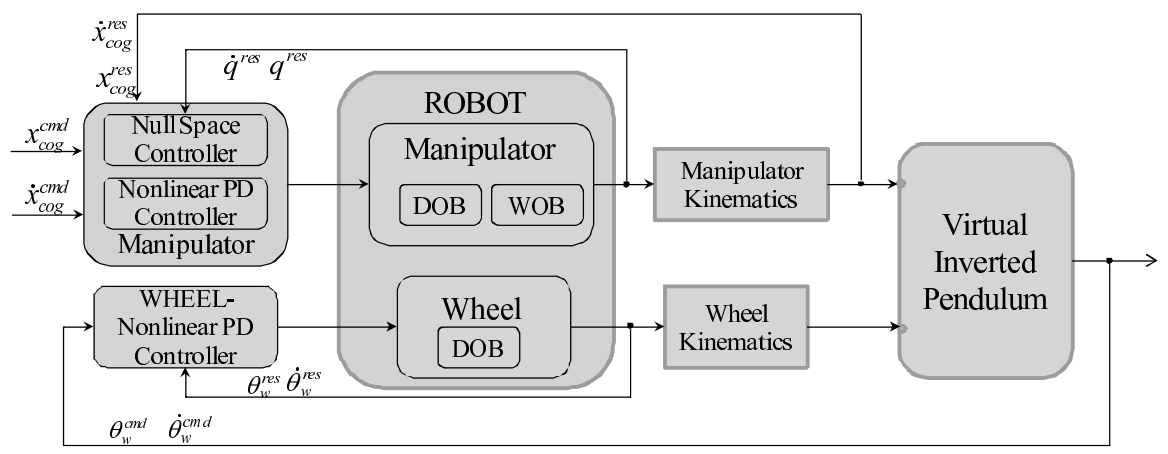

Fig. 6. Full control system 


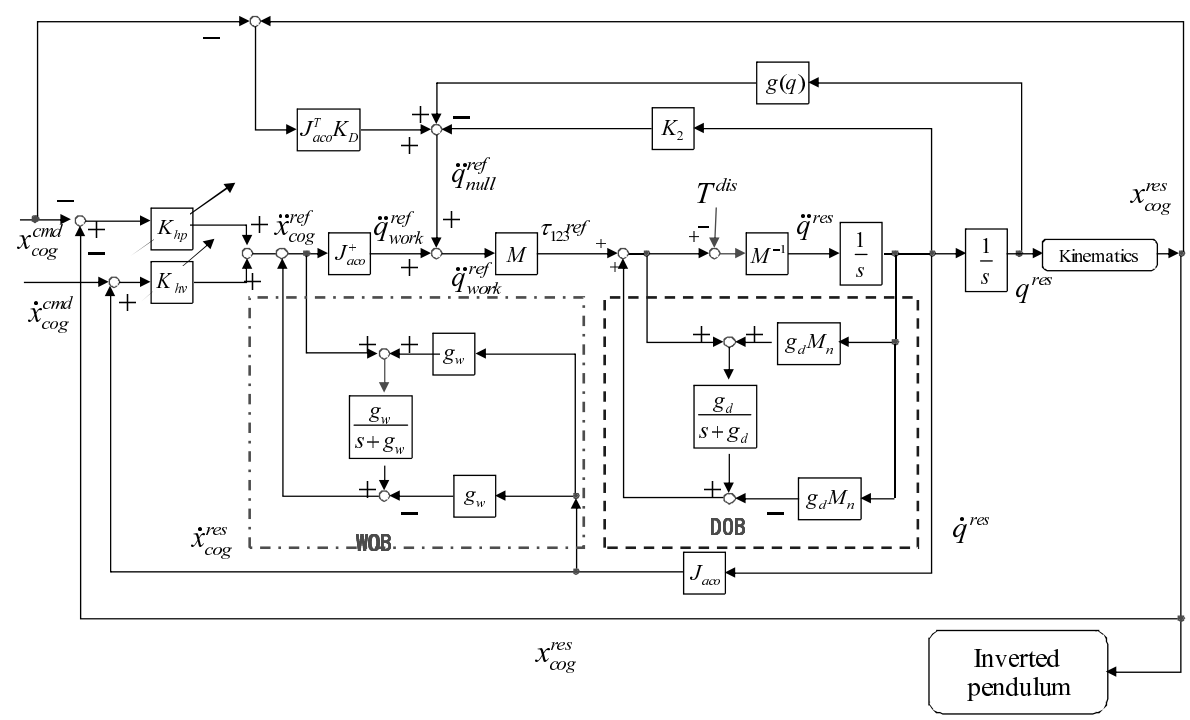

Fig. 7. Block Diagram of Manipulator Control

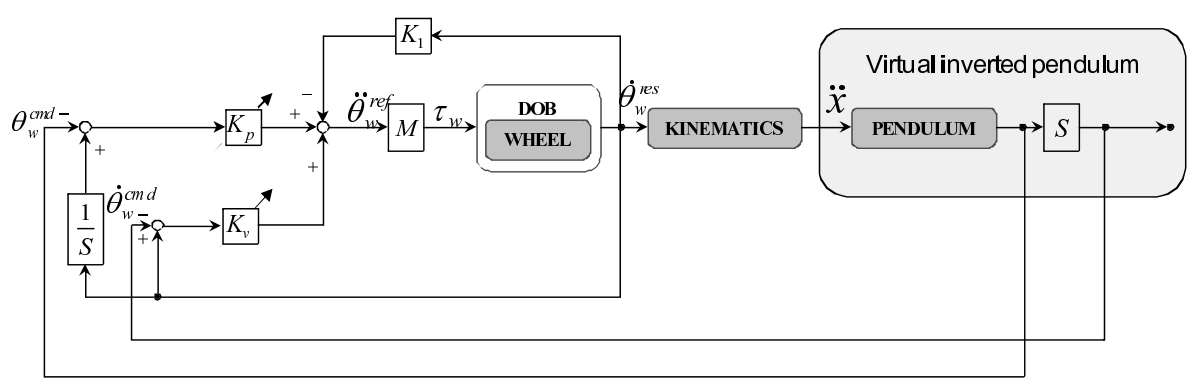

Fig. 8. Diagram of Wheel Controller

controllers are shown in detail. It has been proved that robust motion control can be realized by using disturbance observer ${ }^{(10)}$. Hence, disturbance observer is implemented for for each and every manipulator motor and construction is shown in Fig. 7.

Since wheels are controlled to achieve stable body position, wheel command is generated refering to the inverted pendulum model. Nonlinear PD controller generates the desired acceleration reference. Description of wheel controller with inverted pendulum model is shown in Fig. 8. Estimated disturbance torque is obtained from the velocity reference and current reference. It includes external disturbances, internal forces, inertia change, current fluctuation, Coulomb friction and viscous friction ${ }^{(17)}$.

\section{Experiment Results}

Experiment was carried out to check the validity of proposed method by using the mobile manipulator shown in Fig. 1. Robot details are shown in Table 1. Total weight of two wheel mobile manipulator is $32.4 \mathrm{~kg}$. Experment parameters are shown in Table 2 and Table 3.

Initially, experiment was carried out with conventional PD controller and data was collected. Thereafter, nonlinear PD controller was applied and experiment was repeated in identical conditions. Two wheel mobile manipulator was moved in straightforward direction in both occasions. Fig. 9 shows the robot trajectory in both situations. Continuous line indicates the result with nonlinear PD controller and dash line shows the robot trajectory with conventional PD controller. Robot
Table 1. System parameter used in Experiment

\begin{tabular}{|c|c|c|c|}
\hline Description & Mass $\mathrm{kg}$ & Description & Length $m$ \\
\hline Mass of a wheel & 8.5 & Radius & 0.2 \\
\hline Mass of the body & 13.0 & Length of the link 0 & 0.125 \\
\hline Mass of the link 1 & 1.06 & Length of the link 1 & 0.2 \\
\hline Mass of the link 2 & 0.84 & Length of the link 2 & 0.2 \\
\hline Mass of the link 3 & 0.5 & Length of the link 3 & 0.18 \\
\hline
\end{tabular}

Table 2. Experiment parameter

\begin{tabular}{|c|c|c|}
\hline Description & Notation & value \\
\hline Position gain of wheel command generation & $K_{p d}$ & 10.5 \\
\hline Velocity gain of wheel command generation & $K_{v d}$ & 13.2 \\
\hline Constant - wheel nonlinear PD controller & $K_{0 p}$ & 850.0 \\
\hline Maximum shift - wheel nonlinear PD controller & $K_{1 p}$ & 100.0 \\
\hline Constant - COG nonlinear PD controller & $K_{0 h p}$ & 800.0 \\
\hline Maximum shift - COG nonlinear PD controller & $K_{1 h p}$ & 50.0 \\
\hline
\end{tabular}

Table 3. Experiment parameter

\begin{tabular}{|c|c|}
\hline Constant value, $\zeta_{w}$ & 1.0 \\
\hline Constant value, $\zeta_{m}$ & 1.0 \\
\hline Constant value, $\alpha$ & 2.0 \\
\hline Diagonal of matrix, $K_{1}$ & $(10,10)$ \\
\hline Diagonal of matrix, $K_{2}$ & $(60,60,60,60)$ \\
\hline Diagonal of matrix, $K_{D}$ & $(10,10)$ \\
\hline
\end{tabular}

was appeared to be moving faster with proposed nonlinear PD controller. As well continuous line has shown constant gradient compared to dash line. It could be identified that the velocity fluctuation has been reduced with the proposed method.

Variation of inclination angle of body is shown in Fig. 10. 


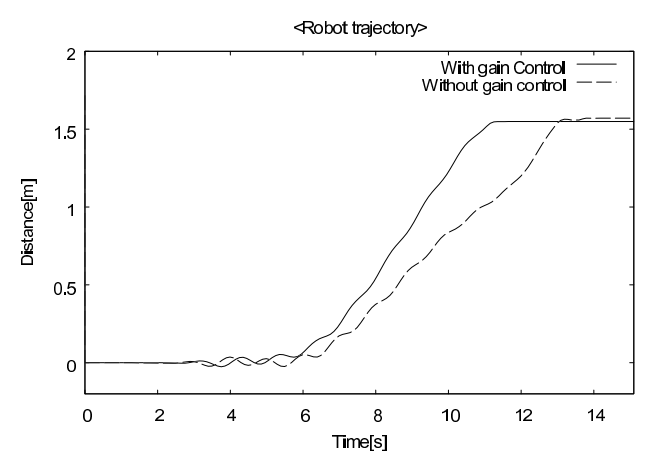

Fig. 9. Robot trajectory

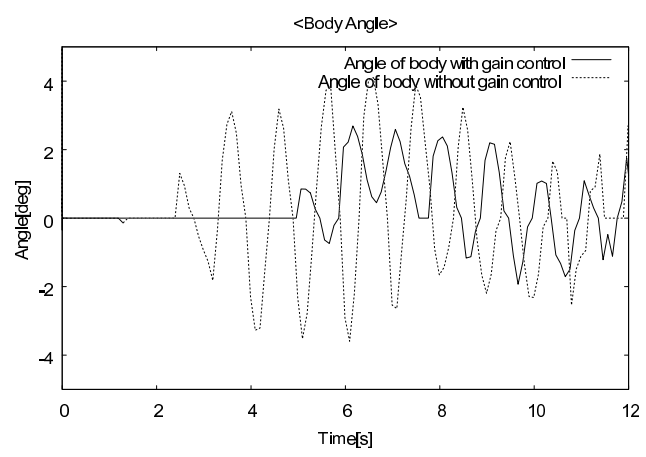

Fig. 10. Variation of Body Angle

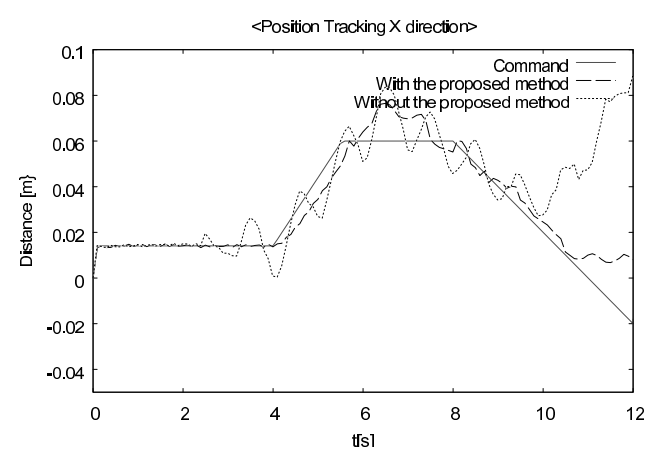

Fig. 11. COG position $-\mathrm{X}$ direction

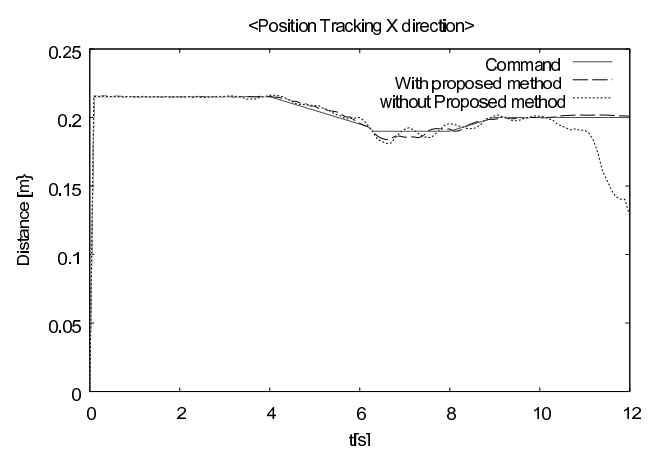

Fig. 12. COG position $-\mathrm{Z}$ direction

Continuous line shows the result with the proposed method while dotted line gives the case of conventional PD controller. It is clear that the amplitude of body angle has been reduced with proposed method. Result proved that body stability has been improved with the nonlinear PD controller.

Fig. 11 and Fig. 12 show the COG positions of the pendulum. Dashed lines show the results with proposed nonlinear PD controller and dotted lines are the results with the con-

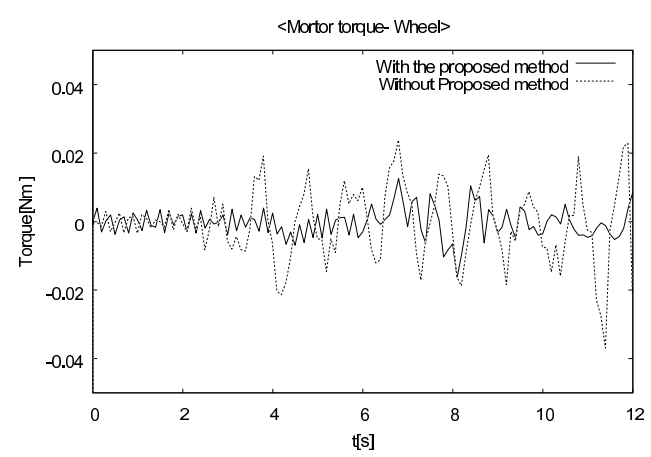

Fig. 13. Torque of wheel motor

ventional PD controller. Continuous lines are commanded COG positions in both graphs. It is evident that controller has followed the commanded value through out the experiment with the proposed method while response COG positions have been deviated from commanded values with the conventional PD controller. Therefore, better COG position tracking was achieved with the proposed nonlinear PD controller.

Fig. 13 is the motor torque of the wheel motor for both occasions. Continuous line illustrates the result with proposed nonlinear PD controller and dotted line gives the result with conventional PD controller. Analyzing these results it is clear that impulse of torque has been reduced with the proposed method. This may be due to the disturbance rejection ability that has in nonlinear PD controller.

\section{Conclusion}

Two wheel mobile manipulator most often includes higher errors due to modeling errors and difficulty of fine tuning. Nonlinear PD control based stability improvement was proposed in this paper. Simultaneous implementation of two nonlinear controller were presented by using mode decomposition. In this research, null space control of manipulator and workspace nonlinear PD control are utilized to improve the performance of the robot system. However, parameter tuning is still very important for obtaining high quality system response. Experiment was carried out to check the validity of the proposed method. Improved results were obtained during experiment. However, fine tuning of some nonlinear terms should be done.

\section{Acknowledgment}

We would like to acknowledge and extend my heartfelt gratitude to Ministry of Education, Culture, Sports, Science, and Technology (MEXT), Japan and KEIO university, Japan for jointly support of this work.

(Manuscript received Aug. 1, 2007,

revised Feb. 22, 2008)

\section{References}

( 1 ) P.R. Ouyang and W.J. Zhang: "Development of an Adaptive Learning PD Control for Robotic System Applications", Control Applications, 2004. Proceedings of the 2004 IEEE International Conference on Vol.1, No.2-4, pp.394-399 (2004-9)

( 2 ) Y. Xu, J.M. Hollerbach, and D. Ma: "A nonlinear PD controller for force and contact transient control", Automatic Control, IEEE Trans. Vol.25, No.3, pp.468-474 (1980-6) 
( 3 ) J. Luh, M. Walker, and R. Paul: "Resolved-acceleration control of mechanical manipulators", The 29th Annual Conference of the IEEE Industrial Electronics Society, 2003. IECON'03. Vol.2, No.2-6, pp.1343-1348 (2003-11)

( 4 ) Y. Xu, D. Ma, and J.M. Hollerbach: "Nonlinear proportional and derivative control for high disturbance rejection and high gain force control", Robotics and Automation, 1993. Proceedings., 1993 IEEE International Conference, No.2-6, pp.752-759 (1993-5)

( 5 ) F. Grasser, A. D’Arrigo, and S. Colombi: "JOE: A Mobile, Inverted Pendulum", IEEE Trans. on industrial electronics, Vol.49, No.1 (2002)

( 6 ) H. Niki and M. Toshiyuki: "An Approach to self Stabilization of Bicycle Motion by Handle Controller", Proceedings of the First Asia International Symposium on Mechatronics (2004-9)

( 7 ) I. Ko, H. Niki, and M. Toshiyuki: "Attitude Control of Bicycle Motion by Steering Angle and Variable COG Control", Industrial Electronics Society, 2005. IECON 2005. 32nd Annual Conference of IEEE 6-10, pp.2065-2070 (2005-11)

( 8 ) H. Abe, T. Shibata, and T. Murakami: "A Realization of Stable Attitude Control in Two Wheels Driven Mobile Manipulator", Second Asia International Symposium on Mechatronics (AISM 2006)

( 9 ) T. Shibata and T. Murakami: "A Null Space Control of Two Wheels Driven Mobile Manipulator Using Passivity Theory", Second Asia International Symposium on Mechatronics (AISM 2006)

(10) K. Ohnishi, M. Toshiyuki, and M. Shibata: "Motion control for Advanced Mechatronics", IEEE/ASME Transactions on Mechatronics, Vol.1, No.1, pp.56-67 (1996-3)

(11) A. Inoue, M. Deng, and T. Tanabe: "Practical Swing-up Control System Design of Cart-type Double Inverted Pendulum", Chinese Control Conference 2006, pp.2141-2146 (2006-8)

(12) C.-I. Huang and L.-C. Fu: "Passivity based control of the double inverted pendulum driven by a linear induction motor", Proceedings of 2003 IEEE Conference on Control Applications, 2003. CCA 2003, Vol.2, No.23-25, pp.797-802 (2003-6)

(13) S. Yasunobu and T. Iwasaki: "Swing up intelligent control of double inverted pendulum based on human knowledge", SICE 2004 Annual Conference, Vol.2, No.4-6, pp.1869-1873 (2004-8)

(14) N. Oda, T. Murakami, and K. Ohnishi: "A force based motion control strategy for hyper-redundant manipulator", Industrial Electronics, Control and Instrumentation, 1997. IECON 97. 23rd International Conference, Vol.3, No.9-14, pp.1385-1390 (1997-11)

(15) T. Murakami, K. Kahlen, and R.W.A.A. De Doncker: "Robust motion control based on projection plane in redundant manipulator", Industrial Electronics, IEEE Transactions, Vol.49, No.1, pp.248-255 (2002-2)
(16) M. Reyhanoglu, A. van der Schaft, N.H. Mcclamroch, I. Kolmanovsky: "Dynamics and control of a class of underactuated mechanical systems", Automatic Control, IEEE Transactions, Vol.44, No.9, pp.1663-1671 (1999-9)

(17) Y. Ha and S. Yuta: "Trajectory tracking control for navigation of selfcontained mobile inverse pendulum", Proceedings of the IEEE/RSJ/GI International Conferenceon, Vol.3, pp.1875-1882 (1994-9)

(18) M. Hatano, M. Minami, T. Ohsumi, and H. Obara: "Modeling of Mobile Manipulators on Irregular Terrain and Evalution of Disturbance Torques", Proceedings of the 2001 IEEE/ASME International Conference on International Conference on Advanced Intelligent Mechatronics Proceedings, pp.12971302 (2001-7)

(19) J.J.E. Slotine and L. Weiping: "Adaptive Manipulator Control: A Case Study", IEEE Trans. on automatic control, Vol.33, No.11 (1988-11)

Pradeep K.W. Abeygunawardhana (Student Member) 1976/8/10

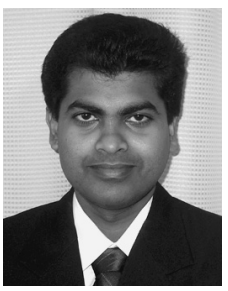
2002 March received the B.Sc.(Eng) specialized in Elecrical Engineering from University of Moratuwa, Sri Lanka. 2006 September received the M.E. degrees from Keio University, Yokohama, Japan. currently a graduate student in Keio University. His research interests are motion control of two wheel robots, Nonlinear control, Wheelchair robot.

Toshiyuki Murakami (Member) 1965/10/05 (M‘93) received the

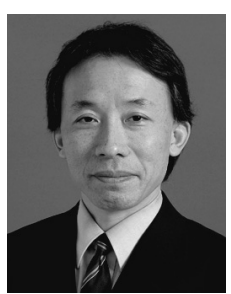
B.E., M.E., and Ph.D degrees in electrical engineering from Keio University, Yokohama, Japan, in 1988, 1990 and 1993, respectively. In 1993, he joined the department of Electrical Engineering, Keio University, where he is currently a professor in the Department of System Design Engineering. From 1999 to 2000, he was a Visiting Researcher with The Institute for Power Electronics and Electrical Drives, Aachen University of Technology, Aechen, Germany. His research interests includes robotics, Intelligent Vehicles, mobile robots and motion control. 